\title{
Examining Eating Habits of Undergraduate Psychology Students
}

\author{
Wendy Salvisberg, Peter Tom, and Sandra Ziolkowski \\ Department of Psychology, University of Alberta
}

\begin{abstract}
The current study examined the relationship between parenting styles, eating and dieting behaviors, and self-esteem in undergraduate psychology students. Standardized assessments of eating and dieting behaviors, parent care and control, and self-esteem were collected from 99 undergraduate psychology students in November 2012. Expected associations of parent care and control with undergraduate students' eating and dieting habits and self-esteem were not found; however, results indicating the influence of mother care and father control on potential eating disorder diagnosis and self-esteem were discovered. Together, these results suggest that more research is needed to explore other characteristics of parenting that may be more related to unhealthy eating and dieting behaviours and self-esteem.
\end{abstract}

\section{PARENT INFLUENCE DURING CHILDHOOD AND ADOLESCENCE}

Parent interactions have a substantial impact on a child's development (Bartholomow, 1990; Bowlby, 1982; Brofenbrenner, 1979; Scaglioni, Salvioni, \& Galimberti, 2008). The way parents interact with their children may influence the development of a child's physical, emotional and mental well-being, which may lead to negative repercussions in adolescence and adulthood. Specifically, family dynamics can powerfully influence the development and maintenance of unhealthy eating/dieting behaviors and lowered self-esteem and in extreme forms, initiate the development of eating disorders (Minuchin, Rosman, \& Baker, 1978; Rodriguez, Novalbos Ruiz, Martinez Nieto, Escobar Jimenez, \& Castro de Haro, 2004; Sira \& White, 2010). Minuchin et al. (1978) proposed a familial model to explain the development of anorexia nervosa. They claimed that the interaction between enmeshment, overprotectiveness, rigidity and lack of conflict resolution within psychosomatic families ultimately contributes to the development of an eating disorder. Researchers have continued to support the link between families characterized by overprotection and excessive control, and anorexia nervosa (Cordero and Israel, 2009; Galloway, Farrow and Martz, 2010; Perry et al., 2006; Shoebridge \& Gower, 2000; Sim et al., 2009; Sira \& White, 2010). Research indicates that eating disturbances are often accompanied by lowered self-esteem (Button, Loan, Davies, \& SonugaBarke, 1998).

\section{Anorexia Nervosa, Bulimia Nervosa, and Eating Disorder Not Otherwise Specified}

According to the Diagnostic and Statistical Manual, Fourth Edition Text-Revised (DSM-IV-TR) (American Psychiatric Association, 2000), an individual meeting the criteria for anorexia nervosa displays behaviours such as refusal to maintain a healthy body weight at or above a minimally normal weight for age and height, amenorrhea, an intense fear of gaining weight, and dis- turbances in self and body image. Individuals with anorexia may be typed as either restricting or bingeeating/purging. Individuals diagnosed with bulimia nervosa may exhibit recurrent episodes of binge eating accompanied by a sense of lack of control over eating, recurrent inappropriate compensatory behaviours to prevent weight gain, and disturbance in self and body image. The binge eating and compensatory behaviours occur, on average, twice a week for 3 months. Bulimia may be classified as either purging or non-purging type. Individuals exhibiting symptoms but not meeting the criteria for any specific eating disorder may be classified as eating disorder not otherwise specified.

\section{Theory on Adolescent Eating and Dieting Behaviours, and Self-Esteem}

The model on psychosomatic families presented by Minuchin et al. (1978) is consistent with Brofenbrenner's ecological systems theory (1979), which theorizes that child development is powerfully influenced by parental behaviors, peer relationships, and societal interactions. The environmental context in which children are raised, most importantly the home setting, helps predict developmental patterns and later emotional development in adolescents (Brofenbrenner, 1979). Parents create environments for children that may foster the development of healthy eating/dieting behaviors, or promote obesity and disordered eating (Scaglioni, Salvioni, \& Galimberti, 2008).

Attachment theorists argue that children develop personal implicit attitudes and beliefs about the self on the basis of parental interactions, specifically based on the responsiveness and sensitivity of primary caregivers (Bartholomow, 1990; Bowlby, 1982). Repeated parentchild interactions thus form the basis for child mental representations of the self in relation to others. Over time and with repeated exposure, how parents respond to children leads to the development of a schema that determines how a child forms and organizes information about the self in relation to other people.

Parenting style can also have a powerful influence on the formation of both explicit and implicit self-esteem 
(Baumrind, 1971; Steinberg \& Morris, 2001). According to Baumrind (1971, p. 22-23), (1) authoritarian parent-child interactions characterized by a lack of nurturance and warmth or (2) permissive parent-child interactions characterized by a lack of control and guidance may lead to lower levels of self-esteem in adolescence. Authoritative parent-child interactions, in comparison, which are characterized with warmth and emotional acceptance, in addition to clearly outlined expectations for acceptable behavior, have been consistently linked with greater adolescent adjustment compared to the two former parenting styles. DeHart, Pelham, and Tennen (2006) further argue that young adults whose parents were consistently nurturing and affectionate reported higher levels of self-esteem compared to parenting styles characterized with lower levels of nurturance and cold behavior.

As healthy self-esteem and eating/dieting behaviors in childhood and adolescence have been found to be crucial indicators of later emotional well-being and mental health, understanding the ways in which parent care and control influences these areas of child development continues to be a crucial area of study. In particular, determining the extent that parents influence eating and dieting behaviours and self-esteem in adolescence is an area of particular importance. In the current study, the effect parent care and control has on undergraduate student's eating/dieting behaviours and self-esteem is investigated.

\section{Research on Parent Care and Control, Adolescent Eating/Dieting Behaviours, and Self-Esteem}

The impact of parent care and control on adolescent eating/dieting behaviours and self-esteem has been investigated in a variety of studies. For example, Renk, McKinney, Klein and Oliveros (2006) conducted a study examining the impact of parent care (i.e., emotional availability) and control (i.e., forms of discipline) on female college students' self-esteem by administering standardized questionnaires. Results of the study suggest that high levels of parent control were inversely correlated with low levels of self-esteem. However, there was no relation between parent care and self-esteem in female college students.

Studies have also investigated the influence of parental control on eating/dieting behaviors independent from self-esteem. For example, Galloway, Farrow and Martz (2010) examined the influence of parent control (i.e., controlled child feeding practices) on college students' eating/dieting behaviours. Parents completed standardized questionnaires of personal parenting styles. Significant positive correlations were found between parent control and current disordered eating patterns in college students.

Cordero and Israel (2009) examined whether perceptions of parent care (i.e., parental acceptance and emo- tional availability) and parent control (i.e., verbal messages relating to eating and exercise regulation) are related to risk factors pertaining to eating pathology (i.e., low self-esteem, body mass index (BMI)) in college women. Expected negative relations between parent care and daughters' eating patterns were not found; however, an element of parent control (i.e., verbal messages relating to eating and exercise regulation) was related to eating pathology. In addition, Cordero and Israel found a negative correlation between self-esteem and unhealthy eating.

Sira \& White (2010) conducted a study investigating the influence of parent care and control on self-esteem, eating/dieting behaviours, and body satisfaction in college students. Based on correlational analyses, high levels of parent control and low levels of paternal care were associated directly with high disordered eating attitude scores among males. In addition, parent control was associated with eating disturbances among males, but not among females. A relationship was found between parental control and other female domains (i.e., body satisfaction), which in turn increased dieting behavior. No direct relationship was found between parent care and eating/dieting behaviours. Self-esteem was directly impacted by parent care and control for both genders.

As demonstrated by the previous studies, many researchers have not found direct relationships between parent care, eating/dieting behaviours, and self-esteem with the exception of Sira \& White (2010). In addition, Perry et al. (2006) investigated the relationship between parent care (i.e., affection and warmth, or coldness and rejection) and control (i.e., encouragement of autonomy/ independence as opposed to strict control with regulation and intrusions), self-esteem and eating behaviours (i.e., eating disturbances) in university students. Unlike many of the previous studies identified, the researchers identified low levels of caring and emotional support and high levels of control to be associated with distorted eating behaviours. Low levels of self-esteem were related to unhealthy eating and dieting behaviours.

It is evident that there are a variety of similarities and differences in the literature investigating the relationship between parent care and control, adolescent eating/dieting behaviours, and self-esteem. Firstly, all of the studies collected data by administering standardized questionnaires to adolescents only; however, Galloway, Farrow and Martz (2010) obtained their results from administering questionnaires to both student and parent participants. Each of these studies used instruments examining respective measures in adolescent or college populations, including the Eating Attitudes Test (EAT-26) (Garner, Olmsted, Bohr, \& Garfinkel, 1982) (Cordero and Israel, 2009; Galloway, Farrow and Martz, 2010; Perry et al., 2006; Sira \& White, 2010), the Rosenberg Self-Esteem Scale (RS-E) (Rosenberg, 1965) (Cordero and Israel, 2009; Renk, McKinney, Klein and Oliveros, 2006) and the Parental Bonding Instrument 
(PBI) (Hall, Peden, Rayens, \& Beebe, 2004) (Perry et al., 2006; Sira \& White, 2010). In addition, all of the studies found a positive relationship between parent control and either eating/dieting behaviours or self-esteem; however, only two found an association between levels of parent care and eating/dieting behaviours, and selfesteem (Perry et al., 2006; Sira \& White, 2010). Overall, the findings of these studies suggest that high levels of parent control (and in two studies, low levels of parent care) are related to future outcomes of adolescents' eating/dieting behaviours and self-esteem. Few studies have examined this relationship in Canadian populations, nor taken into account participant's ethnic background. Also, none of the studies examined whether participants have received or think they could receive an eating disorder diagnosis. As few studies examining non-diagnosed college-age populations have discovered a relationship between parent care and eating/dieting behaviours, this area merits further investigation to explore what extent parent-child interactions influence the development of healthy or disturbed eating and dieting behaviours and self-esteem in university students.

\section{Current Study}

In the current study, parent care and control were assessed by behavioral interactions youth and adolescents have or had with their mother and father, for example, being spoken to in a warm and friendly voice, smiled at frequently, or feeling as if their problems and worries were understood by their parents (parent care). Parent control may be characterized by a lack of autonomy to make personal decisions, feeling babied, or overprotected. Parent care and control was assessed retrospectively, meaning that participants of this study were asked to reflect on their experience during the first 16 years of their life. The purpose of the current study was to examine whether parent care and control relate to eating/dieting behaviours and self-esteem in undergraduate university students. High levels of parent care were expected to positively relate to adolescents' self-esteem, and negatively relate to disordered eating and dieting behaviours. High levels of parent control or overprotection were expected to negatively relate to adolescents' self-esteem, and positively relate to disordered eating/dieting behaviours.

\section{METHOD}

\section{Participants}

Participants included 99 undergraduate students (69\% female) in Psychology 104 and 105 from the University of Alberta in Edmonton, Alberta.
Students studied in a range of faculties with $42 \%$ from the Faculty of Science and 30\% from the Faculty of Arts. Participants represented a range of ethnicities with $60 \%$ being an ethnic minority and 39\% Caucasian/ Canadian/ European. Eighty-seven percent of students identified their parents as having a "married" marital status. Two students indicated that they had received a diagnosis for an eating disorder, and 4 students indicated that in their opinion they could be diagnosed with an eating disorder (compared to 16 indicating they were not sure, and 79 indicating that they could not be diagnosed).

\section{Measures}

Eating and Dieting Behaviours

Students' eating and dieting behaviors were assessed using Part B and C of the EAT-26 (Garner et al., 1982). The EAT-26 is a standardized, self-report 26-item measure that assesses a broad range of symptoms of anorexia and bulimia nervosa. Although initially validated with anorexia patients, the EAT-26 may be used in a non-clinical setting with adolescents and adults to assess risks for disordered eating. Questions on the EAT26 assess potential concerns about dieting, food intake and body weight (i.e., "I eat diet foods," "I am aware of the calorie content of foods that I eat," and "I am occupied with the desire to be thinner"). Each was rated on a 6 -point Likert-scale $(1=$ always to $6=$ never $)$. Respondents were asked to rate frequency of behavior associated with disordered eating and unhealthy diet behaviours (i.e., "In the past 6 months have you gone on binges where you feel that you may not be able to stop?" and "Exercised more than 60 minutes a day to lose or to control your weight?"). Each was rated on a 6 -point Likert-scale $(0=$ never to $5=$ once a day or more). Twenty-five of the items were reverse-coded to create a composite score of disordered eating and dieting behaviors with higher scores indicating higher levels of disordered eating. Multiple psychometric studies have found high internal consistency of the EAT-26 ( $\alpha=$ $.79-.94$ across studies) (Furnham \& Husain, 1999).

\section{Self-Esteem}

The Rosenberg Self-Esteem Scale (RS-E) was used to assess participant's self-esteem (Rosenberg, 1965). The RS-E is a 10-item questionnaire with items answered on a 4 -point Likert-scale $(0=$ strongly agree to $3=$ strongly disagree). Examples of items include, "I feel like I have a number of good qualities," and "I wish I could have more respect for myself." The scale was originally developed with a sample of high school students; however, multiple studies demonstrated good convergent and discriminant validity when used with college students (Hall, Peden, Rayens, \& Beebe, 2004). Five items 
are reverse-coded to create a composite score of selfesteem, with higher scores indicating higher levels of self-esteem.

\section{Parent Care and Control}

Students' retrospective perceptions of parent care and control were measured using the Parental Bonding Instrument (PBI) (Parker, Tuplig, \& Brown, 1979). The PBI is a self-report questionnaire measuring fundamental parental styles as perceived by the participant. Items are answered on a 4 -point Likert-scale $(0=$ very unlike to $3=$ very like) and twelve items are reverse-coded ( $3=$ very unlike to $0=$ very like). Twenty-five questions assessed both maternal and paternal care (i.e., "Mother/ father spoke to me in a warm and friendly voice" and "Mother/father appeared to understand my problems and worries") and control (i.e., "Mother/father tended to baby me" and "Let me go out as often as I wanted"). Results were tallied for both mother and father sections to produce a score for each parent on both dimensions (i.e., mother/father care and mother/father control). Previous studies have confirmed high reliability for each of the scales (paternal care scale $\alpha=.89$; paternal control scale $\alpha=.73$; maternal care scale $\alpha=.86$ and maternal control scale $\alpha=.74$ ) (Sira \& White, 2010).

\section{Procedure}

Students were recruited to the study via the university psychology research pool and received partial course credit for participating in the study. Participants completed 3 structured questionnaires assessing eating/dieting behaviours, levels of self-esteem, and parent care and control. In addition to other measures, the questionnaire package included demographic information of birth month/ year, faculty of study, sex, ethnicity (by continent), and parent marital status. This additional questionnaire included a self-report of whether participants had been diagnosed with an eating disorder, or whether in their opinion they could be diagnosed with an eating disorder. No research was found to support the use of self-reported measures; researchers included the questions out of interest. The researchers guaranteed participants that participation was voluntary, anonymous and confidential. The participants were given an hour to complete the 4 questionnaires.

\section{RESULTS}

\section{Descriptive Analyses}

EAT-26

As shown in Table 1.1, on average, students indicating that they had received a diagnosis for an eating disorder demonstrated higher scores on the EAT-26 ( $M=17.00$, $S D=14.07)$ in comparison to students who indicated that they were not sure whether they could receive a diagnosis $(M=16.75, S D=13.20)$ and receiving no diagnosis $(M=6.66, S D=5.05)$.

These results varied according to ethnic background and gender. Students indicating a European background reported the highest scores on the EAT-26 ( $M=$ $11.75, S D=12.81)$, followed by students of North American background $(M=8.23, S D=9.28)$, Asian background $(M=8.70, S D=7.11)$ and African $(M=$ 8.00, $S D=1.41)$. Females reported higher EAT-26 scores $(M=9.43, S D=9.16)$ compared to males $(M=7.13, S D=6.09)$.

\section{Self-Esteem}

Students indicating that they had received a diagnosis for an eating disorder reported lower self-esteem scores $(M=17.00, S D=5.72)$ in comparison to students who indicated they were not sure whether they could be diagnosed $(M=17.69, S D=4.06)$ and those who indicated they could not be diagnosed $(M=20.59$, $S D=4.44)$.

These results varied according to ethnic background and gender. Students with an Asian background reported lowest levels of self-esteem $(M=19.46, S D=$ 4.38) followed by students of North American background ( $M=20.46, S D=5.01)$, European background $(M=20.62, S D=4.03)$ and African background $(M=23.00, S D=1.41)$. Females reported lower selfesteem scores $(M=19.63, S D=4.75)$ compared to males $(M=20.74, S D=4.05)$. Results are shown in Table 1.2.

\begin{tabular}{lcccc}
\hline \multicolumn{1}{c}{ Variables } & N Mean & SD & Range \\
\hline Diagnosed Eating & Disorder Opinion & \\
No & 79 & 6.66 & 5.05 & $0.00-22.00$ \\
Yes & 4 & 17 & 7.04 & $9.00-38.00$ \\
Not sure & 16 & 16.75 & 3.3 & $4.00-45.00$ \\
Ethnicity by Continent & & & \\
North American & 35 & 8.23 & 9.28 & $1.00-45.00$ \\
European & 8 & 11.75 & 12.81 & $2.00-39.00$ \\
Asian & 54 & 8.7 & 7.11 & $0.00-39.00$ \\
African & 2 & 8 & 1.41 & $7.00-9.00$ \\
\hline
\end{tabular}

TABLE 1.1. Descriptive Statistics of EAT-26 Scores, Diagnosed Eating Disorder Opinion, and Ethnicity by Continent 


\begin{tabular}{lcccc}
\hline \multicolumn{1}{c}{ Variables } & N Mean & SD & Range \\
\hline Diagnosed Eating & Disorder Opinion & \\
No & 79 & 20.59 & 4.44 & $3.00-29.00$ \\
Yes & 4 & 17 & 5.72 & $10.00-24.00$ \\
Not sure & 16 & 17.69 & 4.06 & $8.00-26.00$ \\
Ethnicity by Continent & & & \\
North American & 35 & 20.46 & 5.01 & $3.00-28.00$ \\
European & 8 & 20.62 & 4.03 & $14.00-27.00$ \\
Asian & 54 & 19.46 & 4.38 & $22.00-24.00$ \\
African & 2 & 23 & 1.41 & $3.00-29.00$ \\
\hline
\end{tabular}

TABLE 1.2. Descriptive Statistics of Self-Esteem, Diagnosed Eating Disorder Opinion, and Ethnicity by Continent, and Gender

\section{Parent Care and Control}

Students indicating that they had received a diagnosis for an eating disorder reported lower mother care $(M=19.75, S D=5.52)$ and higher mother control $(M=19.00, S D=9.06)$. Students diagnosed with an eating disorder reported lower levels of father care ( $M=18.25, S D=7.37)$ as well as higher levels of father control $(M=17.75, S D=6.24)$. Participants indicating no eating disorder diagnosis reported highest levels of mother care $(M=29.33, S D=5.52)$ and lowest levels of mother control $(M=14.08, S D=6.49)$. Levels of father control were lowest for participants indicating that they had not been diagnosed with an eating disor$\operatorname{der}(M=10.17, S D=5.77)$. Levels of father care were similar for participants indicating that they were unsure whether they could receive a diagnosis $(M=26.56$, $S D=5.07)$ or no diagnosis $(M=26.32, S D=6.99)$. Results are indicated in Table 1.3. Males reported higher levels of mother care $(M=29.03, S D=5.40)$ than females $(M=28.72, S D=5.48)$; however, females reported higher levels of mother control $(M=14.75$, $S D=6.61)$, father care $(M=26.18, S D=7.10)$ and father control $(M=11.61, S D=6.81)$. Variation in scores by gender is outlined in Table 1.4.

\section{ANOVA \\ EAT-26 and Eating Disorder Diagnosis Opinion}

A $3 \times 1$ (opinion of eating disorder diagnosis $x$ EAT-26 score) between-groups ANOVA design was used to analyze the interaction between whether students felt they could receive a diagnosis of an eating disorder and their reported EAT-26 score. Significant effects were further analyzed using Tukey's HSD $(p<.05)$. A betweensubjects factor analysis revealed a statistically significant interaction between opinion of eating disorder diagnosis and EAT-26 scores, $F(2,96)=15.15, p<.001$.

Participants believing they could be diagnosed with an eating disorder $(M=17.00, S E M=7.04, n=4)$ and participants unsure as to whether they could be diag-

\begin{tabular}{llllll}
\hline Variable I & \multicolumn{2}{l}{ Variable II N } & Mean & SD & Range \\
\hline Parental Bonding & Diagnosed & Eating Disorder Opinion \\
Mother Care & No & 78 & 29.33 & 5.52 & $15.00-36.00$ \\
& Yes & 4 & 19.75 & 3.3 & $16.00-23.00$ \\
& Not sure & 16 & 28.56 & 2.99 & $26.00-35.00$ \\
Mother Control & No & 78 & 14.08 & 6.49 & $3.00-28.00$ \\
& Yes & 4 & 19 & 9.06 & $12.00-31.00$ \\
& Not sure & 16 & 15.75 & 5.52 & $3.00-27.00$ \\
Father Care & No & 78 & 26.32 & 6.99 & $8.00-36.00$ \\
& Yes & 4 & 18.25 & 7.37 & $9.00-27.00$ \\
& Not sure & 16 & 26.56 & 5.07 & $18.00-36.00$ \\
Father Control & No & 78 & 10.17 & 5.78 & $0.00-30.00$ \\
& Yes & 4 & 17.25 & 6.24 & $9.00-24.00$ \\
& Not sure & 16 & 12.44 & 7.96 & $3.00-33.00$ \\
\hline
\end{tabular}

TABLE 1.3. Descriptive Statistics of Parent Care and Control and Diagnosed Eating Disorder Opinion

\begin{tabular}{lllll}
\hline \multicolumn{1}{c}{ Variable I } & Variable II & N & Mean & SD \\
\hline \multirow{3}{*}{ EAT-26 } & Gender & & & \\
& Male & 31 & 7.13 & 6.09 \\
& Female & 68 & 9.43 & 9.16 \\
Self-Esteem & Male & 31 & 20.74 & 4.05 \\
& Female & 68 & 19.63 & 4.75 \\
Mother Care & Male & 31 & 29.03 & 5.4 \\
& Female & 68 & 28.72 & 5.48 \\
Mother Control & Male & 31 & 14.13 & 6.26 \\
& Female & 68 & 14.75 & 6.61 \\
Father Care & Male & 31 & 25.68 & 6.41 \\
& Female & 68 & 25.18 & 7.1 \\
Father Control & Male & 31 & 9.13 & 4.77 \\
& Female & 68 & 11.61 & 6.81 \\
\hline
\end{tabular}

TABLE 1.4. Descriptive Statistics of EAT-26, Self-Esteem, Parental Care and Control and Gender

nosed with an eating disorder $(M=16.75, S E M=3.30$, $n=16$ ) had significantly higher EAT-26 scores than participants not receiving a diagnosis $(M=6.66, S E M=$ $.57, n=79$ ).

A between-subjects factor analysis of EAT-26 scores and ethnicity indicated no significant differences between ethnicity and EAT-26 score.

\section{Self-Esteem and Eating Disorder Diagnosis Opinion}

A $3 \times 1$ (opinion of eating disorder diagnosis $x$ selfesteem) between-groups ANOVA design was used to analyze the interaction between whether students felt they could receive a diagnosis of an eating disorder and their reported self-esteem score. Significant effects were further analyzed using Tukey's HSD $(p<.05)$. 
A between-subjects factor analysis revealed opinion of eating disorder diagnosis interacted to affect selfesteem scores, $F(2,96)=3.82, p<.05$. Participants believing they could not be diagnosed with an eating disorder $(M=20.59, S E M=.50, n=79)$ had significantly higher self-esteem scores than participants unsure as to whether they could receive a diagnosis $(M=17.69$, $S E M=1.02, n=16$ ). However, no significant differences were found between participants believing they could be diagnosed with an eating disorder and selfesteem scores $(M=17.00, S E M=2.86, n=4)$.

A between-subjects factor analysis of self-esteem scores and ethnicity indicated no significant differences between the two variables, $F(3,95)=.70, p>.05$.

\section{Parent Care and Control and Eating Disorder Diagnosis Opinion}

A $3 \times 4$ (opinion of eating disorder diagnosis $x$ mother/father care and control) between-groups ANOVA design was used to analyze the interaction between whether students felt they could receive a diagnosis of an eating disorder and their reported parent care and control score. Significant effects were further analyzed using Tukey's HSD $(p<.05)$.

Analysis revealed opinion of eating disorder diagnosis interacted to affect mother care scores, $F(2,95)=$ $6.63, p<.01$ and father control scores, $F(2,95)=3.14$, $p<.05$. Participants believing they could be diagnosed with an eating disorder $(M=19.75, S E M=1.65$, $n=4$ ) reported significantly lower mother care scores than participants not believing they could be diagnosed $(M=29.33, S E M=.63, n=78)$ and students unsure as to whether they could receive a diagnosis $(M=28.56$, $S E M=.75, n=16$ ). Further analyses found no significant differences in means of reported father control scores and opinions of eating disorder diagnosis.

\section{Independent Sample T-Test}

Gender Differences in EAT-26, Self-Esteem, and Parent Care and Control Scores

A two-tailed independent samples t-test was used to compare EAT-26, self-esteem and reported parent care and control scores in male and female participants. Analyses indicated no significant differences between any of these factors and gender. Marginally significant differences, $p=.07$ were found between female $(M=11.61, S E M=.83, n=67)$ and male $(M=9.13$, $S E M=.86, n=31$ ) ratings of paternal control, with females reporting higher levels of paternal control than males. Results indicated in Table 2.1.

\section{DISCUSSION}

Inconsistent with previous research (Cordero and Israel, 2009; Galloway, Farrow and Martz, 2010; Perry et al., 2006; Renk, McKinney, Klein and Oliveros, 2006; Sira \& White, 2010), the expected positive relations between parent care and self-esteem, and parent control and disordered eating and dieting behaviours were not found in this study. In addition, expected negative relations between parent control and self-esteem, and between parent care and eating/dieting behaviors were also not found. Rather, it was observed that a significantly higher level of maternal care is associated with students believing they could not be diagnosed with an eating disorder, or not sure whether they could receive a diagnosis. Results also indicate students believing they could be diagnosed or were unsure as to whether they could receive a diagnosis reported significantly higher unhealthy eating/dieting behaviors. Students reporting higher levels of self-esteem reported lower levels of unhealthy eating and dieting behavior.

Association between Eating and Dieting Behaviours, Self-Esteem and Parent Care and Control

Relations between parent care and control and selfesteem, and eating/dieting behaviors were not found as expected in research done by Perry et al. (2006), and Sira and White (2010). Previous research found these associations between high levels of control (i.e., encouragement of autonomy/ independence, controlled feeding practices during childhood, discipline and verbal messages relating to eating and exercise regulation) and irregular eating/dieting behaviors, and diminished selfesteem (Renk, McKinney, Klein and Oliveros, 2006); Galloway, Farrow and Martz, 2010); Cordero and Israel, 2009); Sira \& White, 2010; Perry et al., 2006). This challenges Minuchin et al.'s. psychosomatic familial model (1978) that families characterized by overprotectiveness contributes to the development of unhealthy eating and dieting patterns symptomatic of eating disorders. It seems plausible to expect heightened parental control may contribute to the development of unhealthy eating and dieting patterns, and diminished self-esteem, as meticulous behavior surrounding exercise and food are characteristic of developing eating disorders. A possible explanation for the lack of association found may be that participants may have had variable interpretations of parental care and control, and depending on the familial context, the parent care and control variables outlined in the questionnaire may not be applicable to participants. However, females reported marginally higher levels of paternal control than males. This may indicate that excessive paternal control disables the development of autonomy and independence in adolescent females, in turn triggering unhealthy eating and dieting behavior (Sira \& White, 2010). Also, as suggested by Perry et 


\begin{tabular}{lccccccccccc}
\hline & \multicolumn{3}{c}{ Male } & \multicolumn{4}{c}{ Female } & \multicolumn{3}{c}{$\begin{array}{c}\text { Levene's Test } \\
\text { (Eq. variances assumed) }\end{array}$} & $\begin{array}{c}\text { Samples T-Test } \\
\text { (2-tailed) }\end{array}$ \\
\hline Variable & $\mathbf{n}$ & $\mathbf{M}$ & SD & $\mathbf{n}$ & $\mathbf{M}$ & SD & F & Sig. & t & Sig. \\
Eat-26 & 31 & 7.13 & 6.09 & 68 & 9.43 & 9.15 & 0.87 & 0.35 & -1.27 & 0.21 \\
Self-Esteem & 31 & 20.74 & 4.05 & 68 & 19.63 & 4.75 & 0.38 & 0.54 & 1.13 & 0.26 \\
Mother Care & 30 & 29.03 & 5.4 & 68 & 28.72 & 5.48 & 0.01 & 0.91 & 0.26 & 0.79 \\
Mather Control & 31 & 14.13 & 6.26 & 67 & 14.75 & 6.61 & 0.31 & 0.58 & -0.44 & 0.66 \\
Father Care & 31 & 25.68 & 6.41 & 65 & 26.18 & 7.1 & 0.61 & 0.44 & -0.34 & 0.74 \\
Father Control & 31 & 9.13 & 4.77 & 67 & 11.61 & 6.81 & 4.48 & & $.04^{*}$ & -0.18 & 0.07 \\
\hline
\end{tabular}

TABLE 2.1. Independent Samples T-Test of EAT-26, Self-Esteem and Parent Care and Control by Gender

al. (2006), a mediation model may be more effective at explaining the relationship between parent control, eating/dieting behaviours, and self-esteem. For example, parenting variables influence self-image, which in turn impacts eating/dieting behaviours and self-esteem.

\section{Eating Disorder Diagnosis Opinion and Mother Care}

Although not supported by the literature, students reporting that they believe they could not or were not sure whether they could be diagnosed with an eating disorder also reported higher levels of mother care. These results indicate that a caring mother may act as a protective factor from developing unhealthy eating and dieting behaviors. Adolescents who view their mother as a source of emotional support and warmth may in turn adopt a healthy lifestyle characterized by regular food intake and exercise. The lack of findings between paternal warmth and healthy eating patterns may be attributed to the authoritative role that a father plays within a family. A father may take it upon himself to act as a leader and primary provider of the family, and as a result not exhibit as much warmth as a mother. Children, in turn, may not feel comfortable turning to their father as a source of emotional support. These speculations, however, may vary by individual families.

\section{Eating Disorder Diagnosis Opinion, Unhealthy Eating and Dieting Behaviours, and Self-Esteem}

Results indicate that students thinking they could be diagnosed with an eating disorder reported higher levels of unhealthy eating/dieting behavior than individuals thinking they could not be diagnosed. What is interesting to note is that individuals reporting being unsure as to whether they could be diagnosed also reported high levels of unhealthy eating/dieting behavior. This indicates that individuals unsure of deserving diagnosis are equivalent to those sure of deserving diagnosis, and exhibit behaviors symptomatic of a potential eating disorder. Students indicating that they could not receive a diagnosis reported higher levels of self-esteem than those who were not sure. This indicates that these students engaging in normal eating/dieting behaviors feel positively about themselves; however, once unsure as to whether a diagnosis could be received, this in turn may lower an individual's perception of oneself.

\section{Limitations}

It is important to acknowledge limitations of this investigation. First, the sample size in this study included only 99 students, of which $69 \%$ were female. These implications may impact our ability to generalize our findings to a population other than an adolescent post-secondary population. Other means of collecting parent-child relationship data could have been used, for example, by observing the parent's relationship with their child which would lead all researchers to accurately gauge the dynamic of this relationship and decrease self-report and retrospective reporting biases. As well, a survey reporting parents' relationships with their children could be used in the future.

\section{Conclusion and Future Implications}

The purpose of this current study was to examine whether parent care and control relate to eating/dieting behaviours and self-esteem in undergraduate university students. The expected relations between parent care and control, eating/dieting behaviours, and self-esteem were not found. However, results indicate that higher levels of maternal care are associated with students believing they could not or were not sure whether they could be diagnosed with an eating disorder. Results also indicate students believing they could or were unsure as to whether they could receive a diagnosis reported significantly higher unhealthy eating and dieting behaviours. Finally, students reporting higher levels of self-esteem reported lower levels of unhealthy eating/dieting behavior. These findings provide further evidence that although parent care and control were not associated with eating/dieting behaviors, other parenting variables and attitudes more closely related to the consumption of food and exercise behaviors, may merit attention in research that explores the role of parenting in the developing of eating disorders. This research will contribute to the advancement of knowledge by a) help- 
ing to explore behaviors that parents should or should not be engaging in to help prevent the development of unhealthy eating/dieting behaviors; b) sparking the interest in the research of familial implications in the etiology and course of eating disorders, and c) reinforcing the importance of a family-based therapy program for eating disorders or revealing a need for change in the treatment methods. Parenting behaviors should be considered along with other factors that may be contributing to the development of unhealthy eating and dieting behaviors to ensure that adolescents are presented with the best opportunity to engage in a healthy lifestyle.

\section{REFERENCES}

American Psychiatric Association. (2000). Diagnostic and statistical manual of mental disorders (4th ed., text rev.). Washington, DC: Author.

Bartholomew, K. (1990). Avoidance of intimacy: An attachment perspective. Journal of Social \& Personal Relationships, 7, 147-178.

Baumrind, D. (1971). Current patterns of parental authority. Developmental Psychology Monographs, 4(1, Pt. 2), 1-103.

Bowlby, J. (1982). Attachment and loss (Volume 1: Attachment). London: Hogarth Press

Bronfenbrenner, U. (1979). The ecology of human development: Experiments by nature and design. Cambridge, Massachusetts, USA: Harvard University Press.

Button, E. J., Loan, P., Davies, J., \& Sonuga-Barke, E. J. S. (1998). Self-esteem, eating problems, and psychological well-being in a cohort of schoolgirls aged 15-16: A questionnaire and interview study. International Journal of Eating Disorders, 21(1), 39-47.

Cordero, E. D., \& Israel, T. (2009). Parents as protective factors in eating problems of college women. Eating Disorders, 17, 146-161. 10.1080/10640260802714639

DeHart, T., Pelham, B.W., \& Tennen, H. (2006). What lies beneath: Parenting style and implicit self-esteem. Journal of Experimental Social Psychology, 42, 1-17.

Furnham, A., \& Husain, K. (1999). The role of conflict with parents in disordered eating among British Asian females. Social Psychiatry \& Psychiatric Epidemiology, 34, 498505.

Galloway, A. T., Farrow, C. V., \& Martz, D. M. (2010). Retrospective reports of child feeding practices, current eating behaviours, and BMI in college students. Obesity, 18, 1330-1335. doi:10.1038/oby.2009.393

Garner, D. M., Olmsted, M. P., Bohr, Y., \& Garfinkel, P. E. (1982). The eating attitude test: psychometric fea- tures and clinical correlates. Psychological Medicine, 12,871878 .

Hall, L. A., Peden, A. R., Rayens, M. K., \& Beebe, L. H. (2004). Parental bonding: A key factor for mentalhealth of college women. Issues in Mental Health Nursing, 25, 277291.

Minuchin, S., Rosman, B. L., \& Baker, L. (1978). Psychosomatic families: Anorexia nervosa in context. Cambridge, Massachusetts, USA: Harvard University Press.

Parker, G., Tuplig, H., \& Brown, L. B. (1979). A parental bonding instrument. British Journal of Medical Psychology, 52, 110.

Perry, J. A., Silvera, D. H., Neilands, T. B., Rosenvinge, J. H., \& Hanssen, T. (2007). A study of the relationship between parental bonding, self-concept and eating disturbances in Norwegian and American college populations. Eating Behaviours, 9, 13-24.

Renk, K., McKinney, C., Klein, J., \& Oliveros, A. (2005). Childhood discipline, perceptions of parents, and current functioning in female college students. Journal of Adolescence, 29, 73-88. doi:10.1016/j.adolescence.2005.01.006

Rodriguez, M. A., Novalbos Ruiz, J. P., Martinez Nieto, J. M., Escobar Jimenez, L., \& Castro de Haro, A. L. (2004). Epidemiological study of the influence of family and socioeconomic status in disorders of eating behaviour. European Journal of Clinical Nutrition, 58(6), 846-852.

Rosenberg, M. (1965). Society and the adolescent selfimage. Princeton, NJ: Princeton, University Press.

Scaglioni, S., Salvioni, M., \& Galimberti, C. (2008). Influence of parental attitudes in the development of children eating behavior. British Journal of Nutrition, 99(1), S22- 25. doi: 10.1017/S0007114508892471

Shoebridge, P. J., \& Gower, S. G. (2000). Parental high concern and adolescent-onset anorexia nervosa: A case-control study to investigate direction of causality. British Journal of Psychiatry. 176, 132-137. doi: 10.1192/bjp.176.2.132

Sim, L. A., Homme, J. H., Lteif, A. N., Vande Voort, J. L., Schak, K. M., \& Elingson, J. (2009). Family functioning and maternal distress in adolescent girls with anorexia nervosa. International Journal of Eating Disorders, 42, 531-539.

Sira, N. \& White, C. P. (2010). Individual and familial correlates of body satisfaction in male and female college students. Journal of American College Health, 58(6), 507-514.

Steinberg, L. \& Morris, A.S. (2001). Adolescent development. Annual Review of Psychology, 52, 83-110. 